PAPER

\title{
Justice and the NICE approach
}

\author{
Richard Cookson
}

\section{Correspondence to}

Dr Richard Cookson, Centre for Health Economics, University of York, York Y010 5DD, UK; richard.cookson@york.ac.uk

Received 5 September 2014 Revised 26 September 2014 Accepted 3 October 2014
CrossMark

To cite: Cookson R. J Med Ethics 2015;41:99-102.

\section{ABSTRACT}

When thinking about population level healthcare priority setting decisions, such as those made by the National Institute for Health and Care Excellence, good medical ethics requires attention to three main principles of health justice: (1) cost-effectiveness, an aspect of beneficence, (2) non-discrimination, and (3) priority to the worse off in terms of both current severity of illness and lifetime health. Applying these principles requires consideration of the identified patients who benefit from decisions and the unidentified patients who bear the opportunity costs.

\section{INTRODUCTION}

What is it to do good medical ethics? An uncharitable reader of the philosophical medical ethics literature might be forgiven for answering, 'come up with an absurdly unrealistic example, and use it to argue for an absurdly counter-intuitive conclusion.'

This flippant remark is, of course, grossly unfair. Yet replace 'absurdly' with 'appropriately', and it describes two important virtues of ethical thinking. Unrealistic examples can help clarify our thinking by placing the essential features of an ethical problem into sharp focus. And common sense intuitions can lead us astray. Our moral psychology was shaped by evolution during millions of years of human prehistory living in small hunter-gatherer tribes. The resulting intuitions may sometimes lead us astray in today's much larger and more technologically advanced societies. Rather than resting content with 'fast thinking' intuitions, therefore, it behoves us to engage in some 'slow thinking' to arrive at more considered ethical judgements. ${ }^{12}$

The medical ethics literature pays close attention to technological advances in medicine and how they raise new ethical challenges. However, it pays less attention to another important difference between hunter-gatherer and modern societies: size. Hunter-gather tribes rarely contained more than around 150 people-the 'Dunbar number', above which humans and primates find it hard to handle social relationships ${ }^{3}$ - and everyone knew each other. By contrast, modern societies are vast and impersonal. National governments routinely make decisions that influence thousands, millions and in some cases even hundreds of millions of fellow citizens who do not know each other.

Medical ethicists spend a lot of time discussing hypothetical examples involving a small number of identified patients. In this essay, I want to focus on population level decisions involving a large number of unidentified patients. My focus is not on clinical decisions about particular patients, but on policy decisions by healthcare managers and policymakers about the institutional, regulatory and financial environment within which such clinical decisions are made.

A paradigmatic example is the National Institute for Health and Care Excellence (NICE). NICE produces guidance on the use of healthcare technologies within the single payer, universal and comprehensive National Health Service (NHS) in England and Wales. However, NICE has no control over the size of the tax-funded NHS budget or how local NHS organisations manage their budgets. When NICE recommends the use of a cost-increasing healthcare technology for one particular group of patients, therefore, it is implicitly recommending the displacement of expenditure on unknown alternative uses of NHS money for unknown other patients.

What principles of justice should govern such decisions about the allocation of scarce healthcare resources? I will sketch out the 'NICE approach' to this question-or, rather, my own interpretation of it-and argue that it embodies 'good' rather than 'bad' medical ethics.

The starting point for NICE is the procedural justice framework that bioethicist Norman Daniels has dubbed 'accountability for reasonableness', with its four requirements of: (i) publicly accessible decisions and the rationales for them, (ii) reasonableness of rationales in the sense both of giving reasons and applying relevant principles, (iii) the possibility of challenge through appeal and of revision of decisions and (iv) the presence of mechanisms to ensure that the foregoing requirements are met. ${ }^{4}$ Within this framework, NICE focuses on three substantive principles of justice: (1) costeffectiveness, (2) non-discrimination and (3) priority to the worse off. ${ }^{5}$ I will start with cost-effectiveness.

\section{COST-EFFECTIVENESS}

Should opportunity costs for large numbers of unidentified people be taken into account in healthcare priority setting decisions? NICE thinks the answer is an unequivocal 'yes'. It accounts for opportunity costs to unknown patients in exactly the same way that it accounts for benefits to the known patients who use the healthcare technology under consideration. It values these opportunity costs in terms of the expected impact on the length and quality of people's lives using the 'quality adjusted life year' (QALY). One QALY represents 1 year of life in full health, half a QALY represents a year of life in 50\% health and so on. NICE currently estimates that a reduction of $£ 20000$ pounds of NHS expenditure on unknown NHS activities benefiting unknown patients will typically 
have an opportunity cost of one QALY. This is not far off the current best estimate of this 'cost per QALY threshold value' of $£ 18000$, based on real data on variation in NHS expenditure and outcomes between subnational administrative areas (formerly known as 'Primary Care Trusts' when the data were collected in 2008, and now 'Clinical Commissioning Groups'). ${ }^{6}$ By using this estimate to value opportunity costs, NICE is making the value judgement that each QALY lost by unknown NHS patients has the same value as each QALY gained by the known group of patients who use the healthcare technology under consideration.

This value judgement is a straightforward corollary of the principle of cost-effectiveness that healthcare resources should be used to improve population health. Stewards of the public purse have a duty of 'beneficence' towards all the citizens they serve, to do as much good as possible with scarce public resources. In the context of healthcare, it seems reasonable to interpret this as a duty to improve population health. This seems to be the UK government's interpretation since it charges both NICE and the NHS with the objective of improving population health.

How should 'population health' be measured? The value judgement made by NICE is that units of health should simply be added up across different citizens, on the basis that 'a QALY is a QALY is a QALY'. It is thus assumed that a year or a day or indeed an hour of life in full health has the same value, no matter who lives it. A QALY thus the same value no matter whether it is lived by a known or an unknown group of citizens, no matter whether it is seen as a gain or a loss from the reference point of the current situation, and no matter how many healthy years are gained or lost in total compared with the current situation.

That value judgement is illustrated in figure 1 . The horizontal $\mathrm{x}$-axis shows the health opportunity cost in terms of the total number of QALYs lost by unknown patients. The vertical y-axis shows the total value of those health opportunity costs, according to NICE. It is a $45^{\circ}$ straight line, reflecting the NICE value judgement that each QALY is worth the same.

\section{Psychic numbing}

The NICE approach contrasts sharply with the common sense intuitions embedded in our moral psychology. Drawing on evidence from psychological experiments, as well as the

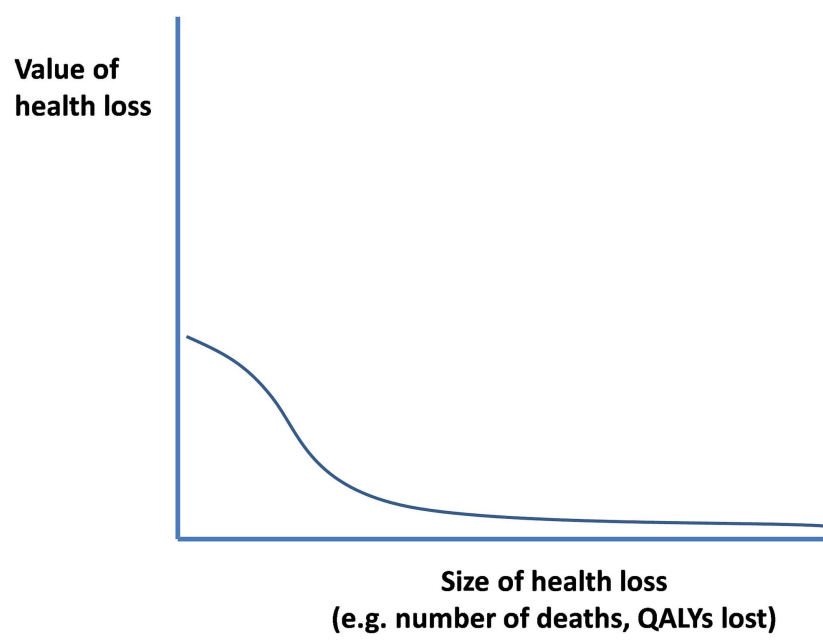

Figure 2 Public concern for health opportunity costs under psychic numbing.

observations of social commentators and our own everyday experience, Paul Slovic ${ }^{7}$ has described the phenomenon of 'psychophysical numbing' and its more extreme cousin of 'psychic numbing'. The psychology evidence typically focuses on health losses framed in terms of numbers of deaths; but the same idea can be applied to any numerical unit of health loss, including QALYs.

Psychophysical numbing involves diminishing marginal concern for health losses. In that case, we care a lot about a single unit of health loss, but then a bit less about the next one, a bit less still about the next one and so on. This implies that our concern for an opportunity cost of 1000 QALYs is not much greater than our concern for an opportunity cost of 1 QALY, and nowhere near 1000 times greater as the NICE approach implies. Psychic numbing is more extreme, and involves diminishing total concern for health losses, as illustrated in figure 2 . In that case, we care more about a health loss of 1 QALY to a single identified person than about a health loss of 1 QALY each to 1000 unidentified people.

Figure 3 compares the NICE approach with common sense ethical intuitions. When thinking about health opportunity costs to large numbers of unknown people, our common sense

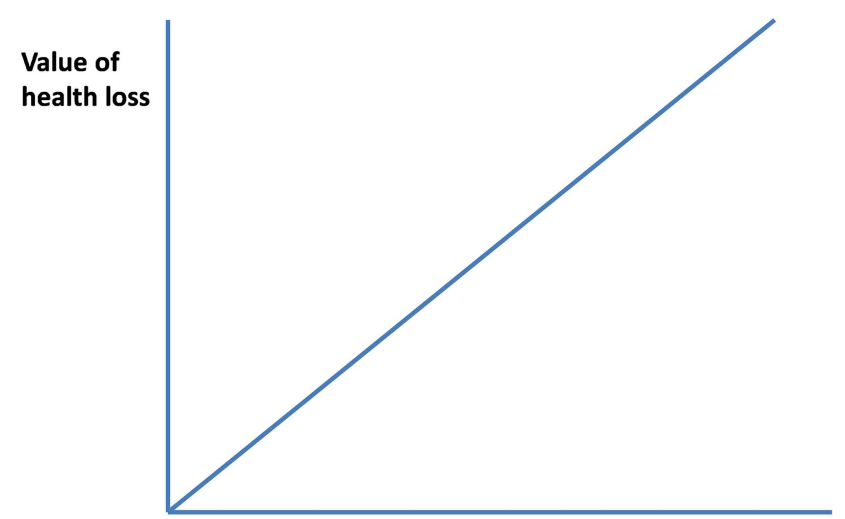

Size of health loss

(e.g. number of deaths, QALYs lost)

Figure 1 National Institute for Health and Care Excellence's valuation of health opportunity costs.

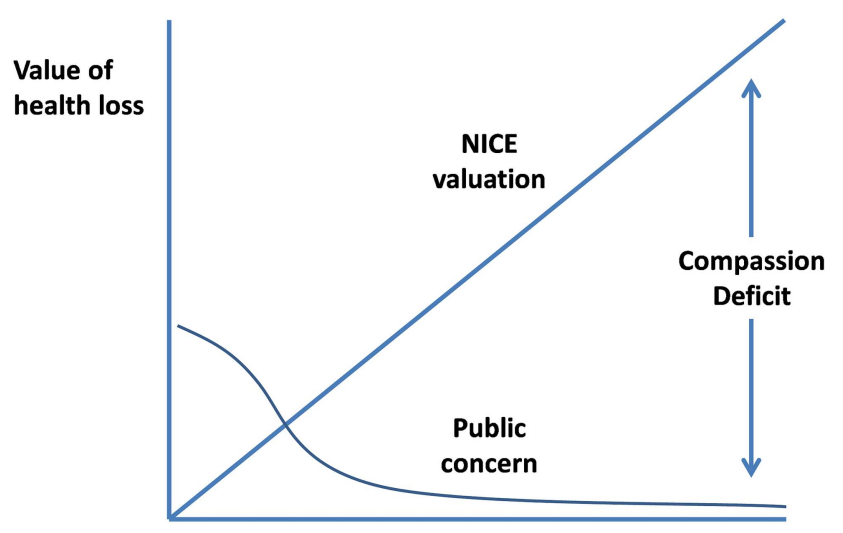

Size of health loss

(e.g. number of deaths, QALYs lost)

Figure 3 Compassion deficit-National Institute for Health and Care Excellence (NICE) valuation versus public concern under psychic numbing. 
intuitions result in what we might call a 'compassion deficit'. We are capable of feeling a high degree of compassion about a health loss to a single identified person, but we are not capable of feeling thousands of times more compassion about health losses to thousands of unidentified people. Indeed, because our instincts evolved to feel compassion towards identifiable individuals, we tend to feel more compassion for a single known individual than thousands of unidentified individuals.

\section{Crude utilitarianism?}

Does the NICE approach imply a crude utilitarianism that favours inhumane acts, for example, the callous neglect of severely ill patients who fail the cost-effectiveness test? Or 'Brave New World' style happiness pills for all, coupled with 'Logan's Run' style involuntary euthanasia for the over 30s? Of course not! For one thing, QALYs measure health and longevity, not happiness. Happiness pills do not necessarily increase health and longevity - for that, you need health and longevity pillsand involuntary euthanasia would reduce longevity. So the NICE approach is not 'utilitarian', at least not in the classical Benthamite sense of maximising the sum total of all the happy experiences in the universe.

The NICE approach is not crude, either. NICE is pluralistic about principles of justice and does not endorse the value judgement that sum total health impact is the only relevant ethical consideration. Cost-effectiveness is just one important consideration that NICE takes into account when reaching its decisions -not the only one. NICE does not set a rigid cost-effectiveness cut-off point for recommending healthcare technologies; it merely sets a cost-effectiveness range beyond which a positive recommendation requires particularly strong and careful justification through wider considerations. In line with the 'accountability for reasonableness' framework described above, NICE formulates its recommendations through a deliberative process which involves diverse stakeholders and takes into account a wide range of considerations.

\section{NON-DISCRIMINATION}

The principle of non-discrimination acts as an ethical constraint on the principle of cost-effectiveness. Discussions about costeffectiveness and discrimination in the medical ethics literature often focus on clinical decisions about particular individual patients. This is potentially misleading because in practice costeffectiveness calculations based on QALYs are only ever used to inform population level decisions involving large numbers of unidentified patients. So here I want to consider a hypothetical example of potential disability discrimination that is more akin to the kinds of population level decisions made by NICE. Imagine NICE is faced with a new life extending treatment for stomach cancer that is borderline cost-effective in the full patient population. Further, imagine that the treatment has the same probability in all patents of a good biomedical response in terms of shrinking the tumour and restoring patients to their previous level of health and expected longevity. But now imagine it is not cost-effective for a subpopulation of severely disabled individuals, because their previous level of health and expected longevity are lower than average. The principle of cost-effectiveness thus suggests recommending that the treatment should be funded for most patients, but not for severely disabled patients. However, that would be an act of invidious discrimination against the disabled; it would generate political outrage; and it might also be contrary to European legislation on human rights. So NICE would not do it. Indeed, I doubt NICE advisory committees ever find themselves having to consider a cost-effectiveness argument for discriminating against the disabled, since subgroup analyses of cost-effectiveness based on severe disability are rarely if ever performed. NICE often undertakes subgroup analysis on suitable patient and treatment characteristics in pursuit of cost-effective ways of funding a particular healthcare technology, but it does not strive officiously to find opportunities for disability discrimination.

\section{PRIORITY TO THE WORSE OFF}

Priority to the worse off in terms of severity of illness is a principle of justice that reflects policy concern to distribute healthcare according to need, insofar as severity of illness is an important component of need. In pursuit of accountability, NICE has recently proposed two ways of measuring the severity or 'burden' of illness. First, the absolute shortfall from normal healthy life expectancy. This is the difference between the years of life in full health that the average person would expect, given their age and sex, and what the patient can expect given the severity of their disease. Second, the relative shortfall. This is the absolute shortfall expressed as a percentage of normal healthy life expectancy. Absolute shortfall tends to be larger in younger patients who have longer left to live and so will tend to suffer a larger absolute shortfall from any given disease in terms of years of healthy life lost.

Importantly, NICE recognises that NHS patients who bear the health opportunity costs of its decisions may also be severely ill. So it compares severity of illness not against healthy individuals with 'zero' severity of illness but against a benchmark severity of illness for the typical NHS patient. However, NICE has not gone so far as to specify a formula for giving greater weight to QALY gains for patients with more severe illnesses. This may be wise, given the complexity of judgements about justice and structural differences between principles of beneficence versus priority to the worse off. ${ }^{8}$ Instead, NICE will continue to make these nuanced judgements on a case by case basis, through a deliberative process.

A quite different way of defining the 'worse off' is in terms of lifetime health, rather than current severity of illness. Priority to the worse off in terms of lifetime health is a principle of justice that reflects policy concern to reduce social inequalities in longevity and health. ${ }^{9}$ In a healthcare context, however, the principle of non-discrimination often acts as an ethical constraint against this principle. For example, the NHS might be able slightly to reduce social inequality in health by funding a treatment only for the poor. Yet this might seem like invidious discrimination against the rich. In my view, therefore, the principle of priority to those with poor lifetime health is typically more relevant in relation to healthcare decisions about improving access to and uptake of preventive healthcare, rather than decisions about whether to fund particular treatments. For example, this principle may be relevant in decisions about whether to locate smoking cessation clinics near affluent or deprived neighbourhoods, or whether to make special additional marketing and outreach efforts to encourage people from socially disadvantaged backgrounds to attend a cancer screening programme.

\section{CONCLUSIONS}

I have argued that, when thinking about population level healthcare decisions, opportunity costs for unidentified fellow citizens are an essential feature of the ethical landscape. Justice concerns 
the distribution of burdens as well as benefits. So in order to take appropriate account of justice, good medical ethics requires careful consideration of the opportunity costs of healthcare decisions and who will bear them. We need to consider the total size of those opportunity costs in order to apply the ethical principle of beneficence or cost-effectiveness. We also need to consider who will bear those opportunity costs in order to apply the ethical principle of priority to the worse off.

Funding RC is funded by a Senior Research Fellowship award from the National Institute for Health Research.

Competing interests None.

Disclaimer The views expressed in this publication are those of the author and not necessarily those of NICE, the NHS, the National Institute for Health Research or the Department of Health.

Provenance and peer review Commissioned; internally peer reviewed.

\section{REFERENCES}

1 Kahneman D. Thinking, fast and slow. 1st edn. New York: Farrar, Straus and Giroux, 2011.

2 Greene JD. Moral tribes: emotion, reason, and the gap between us and them. Penguin Press, 2013.

3 Cozolino L. The neuroscience of human relationships: Attachment and the developing social brain. WW Norton \& Company, 2014.

4 Daniels N. Accountability for reasonableness. BMJ 2000;321(7272):1300-1.

5 Shah KK, Cookson R, Culyer AJ, et al. NICE's social value judgements about equity in health and health care. Health Econ Policy Law 2013;8(2):145-65.

6 Claxton K, Martin S, Soares M, et al. Methods for the estimation of the NICE cost effectiveness threshold. York: University of York, 2013.

7 Slovic P. If I look at the mass I will never act: Psychic numbing and genocide. Judgment Decis Mak 2007;2(2):79-95.

8 Broome J. Fairness versus doing the most good. Hastings Cent Rep 1994;24 (4):36-9.

9 Eyal N, Hurst SA, Norheim OF, et al. Inequalities in health: concepts, measures, and ethics. Oxford: Oxford University Press, 2013. 